\title{
Influencia de Los Factores Ambientales En La Producción Relativa de Tricotecenos por Fusarium Graminearum
}

\author{
Diana Ramirez Albuquerque (I), Monica Sepulveda (I), Adriana \\ Kolender (I), Andrea Patriarca (I), Virginia Fernandez Pinto (I) \\ (I) FCEN UBA - Departamento de Quimica Organica (Ciudad Universitaria Buenos Aires \\ Argentina)
}

\section{Resumo}

Una de las principales enfermedades que afectan el cultivo del trigo es la 'Fusariosis de la Espiga' (FET). La infección ocurre principalmente cuando se dan las condiciones favorables de humedad y temperatura durante la antesis o floración, siendo F. graminearum el agente causal más comun. Se identificaron tres quimotipos entre los aislamientos de F. graminearum, uno de los cuales produce deoxinivalenol (DON) y 3-acetildeoxinivalenol (3ADON), otro productor de DON y 15-acetildeoxinivalenol (15ADON) y un tercer quimiotipo que produce nivalenol (NIV). El quimiotipo 15ADON es predominante en Argentina, aunque también se han encontrado aislamientos pertenecientes al quimiotipo $3 \mathrm{ADON}$ y otros que producen cantidades semejantes de ambos acetilderivados. Análisis moleculares y de producción de micotoxinas demostraron que el quimiotipo 3ADON está desplazando rápidamente al quimiotipo $15 \mathrm{ADON}$ en poblaciones de $\mathrm{F}$. graminearum del oeste de Canadá y que los aislamientos pertenecientes al quimiotipo $3 \mathrm{ADON}$ producían más DON que los aislamientos pertenecientes al quimiotipo $15 \mathrm{ADON}$. Para poder diseñar estrategias adecuadas de control de la enfermedad, es importante determinar cómo influyen los factores climáticos en la preponderancia de uno u otro quimiotipo. El objetivo de este trabajo fue estudiar la influencia de la temperatura y la aw sobre el la producción de uno u otro acetil derivado utilizando una cepa de Fusarium graminearum productora de DON, 3- y

\footnotetext{
Referência:

Diana Ramirez Albuquerque, Monica Sepulveda, Adriana Kolender, Andrea Patriarca, Virginia Fernandez Pinto.Influencia de Los Factores Ambientales En La Producción Relativa de Tricotecenos por Fusarium Graminearum. In: Anais do 12 Congresso Latinoamericano de Microbiologia e Higiene de Alimentos - MICROAL 2014 [= Blucher Food Science Proceedings, num.1, vol.1]. São Paulo: Editora Blucher, 2014. 
15-ADON. Los ensayos se realizaron sembrando la cepa en un medio agarizado con $2 \%$ de trigo, con aw ajustada a 0.95 y 0.99 con glicerol. Se incubó a $10,15,20,25$ y $30^{\circ} \mathrm{C}$ por $7,14,21$ y 28 días respectivamente. Las toxinas se extrajeron con acetonitrilo/acetato de etilo/agua (50:41:9, v/v) realizándose el clean-up en una columna con $0,7 \mathrm{~g}$ de carbón activado, 0,5 $\mathrm{g}$ de alúmina neutra y $0,3 \mathrm{~g}$ de celite. La detección de tricotecenos se realizó por CG con detector de captura de electrones. Los factores ambientales tuvieron gran influencia en la síntesis relativa de los derivados acetilados, siendo esta influencia más marcada en el caso de la temperatura ya que los maximos de producción de DON $(31,174 \mu \mathrm{g} / \mathrm{g}$; aw $0.99 ; 14$ días) y $3 \mathrm{ADON}(61,875 \mu \mathrm{g} / \mathrm{g}$; aw $0.99 ; 21$ dias $)$ se observaron a $30^{\circ} \mathrm{C}$ y los de $15 \operatorname{ADON}(34,962 \mathrm{ug} / \mathrm{g}$; aw $0.99 ; 7$ días $)$ a $10^{\circ} \mathrm{C}$.

Palavras-Chave: Fusarium graminearum, Tricotecenos, aw, Temperatura Agência de Fomento: 